\title{
Ambulatory arterial stiffness index is increased in obese children
}

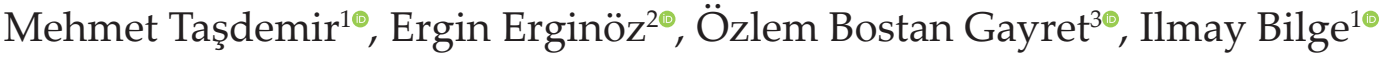 \\ ${ }^{1}$ Division of Pediatric Nephrology, ${ }^{2}$ Department of Pediatrics, Koç University School of Medicine, İstanbul; ${ }^{3}$ Department of Pediatrics, \\ Health Sciences University, Bağcllar Training and Research Hospital, İstanbul, Turkey.
}

\begin{abstract}
Background and objectives. One way to measure arterial stiffness is the ambulatory arterial stiffness index (AASI), which is the relationship between diastolic and systolic ambulatory blood pressure (BP) over 24-hours.

Methods. We studied the difference in AASI between obese and lean children. AASI was calculated from 24hour ambulatory blood pressure monitoring in 53 obese children (33 girls) and compared with age-matched 42 healthy subjects (20 girls). Hypertension was defined according to the criteria of the American Heart Association. To evaluate inflammation, the blood level of high-sensitive C-reactive protein was measured.
\end{abstract}

Results. The mean age was $10.6 \pm 2.83$ years in obese children and $11.3 \pm 3.17$ years in healthy subjects. Hypertension was determined in three (5.6\%) obese children. The median heart rate-SDS, pulse pressure and blood pressure values did not differ between the two groups. The mean AASI was significantly higher in obese children compared to healthy subjects $(0.42 \pm 0.15$ vs. $0.29 \pm 0.18, \mathrm{p}<0.001)$. AASI significantly correlated with nighttime SBP-SDS, nighttime SBP-load, systolic and diastolic nocturnal dipping, with no independent predictor.

Conclusion. This study confirms that AASI is increased in obese children. AASI calculation is a useful, costeffective, and an easy method to evaluate arterial stiffness. Early detection of increased arterial stiffness can help clinicians come up with preventive measures in the management of patients.

Key words: arterial stiffness, blood pressure measurement, cardiovascular risk, children, obesity.

In recent years, childhood obesity has become an epidemic health problem. The prevalence of obesity is more common in developed countries (16-30\%) compared to underdeveloped countries (6-13\%). ${ }^{1}$ The increase in prevalence of obesity has led to the emergence of comorbidities associated with it. Hypertension is one of the most important comorbidities that is associated with increased body mass index (BMI) in children and its presence in

\footnotetext{
凶 Mehmet Taşdemir

mtasdemir@kuh.ku.edu.tr
}

Received 19th March 2018, revised 3rd June 2018, 20th August 2018, 10th September 2018,

27th November 2018, accepted 4th March 2019.

This study has been presented as a poster at the 50th Anniversary Meeting of the European Society for Pediatric Nephrology (ESPN), 6-9 September 2017, Glasgow, Scotland. adulthood is an important risk factor for early cardiovascular disease (CVD) ${ }^{2-4}$ Cardiovascular $(\mathrm{CV})$ risk factors including hypertension, dyslipidemia, metabolic syndrome, and obesity itself have effects on vascular function and structure such as endothelial dysfunction, increased carotid artery intima media thickness (IMT), and increased arterial stiffness. ${ }^{5,6}$

To evaluate vascular function and structure, different techniques have been used in the past. These include measurements of carotid artery IMT, pulse wave velocity (PWV), and flow mediated dilatation. Arterial stiffness is a measure of vascular elastic behavior which can be altered by different etiologies such as atherosclerosis, hypertension, dyslipidemia and obesity. Decreases in elastic mechanical features of arteries lead to increased arterial stiffness in obese children. ${ }^{7}$ Since measurements of vascular 
structure and function by IMT, PWV, or other ways are required by experienced persons and expensive tools, ambulatory arterial stiffness index (AASI) as a less complex method was developed in 2006.

AASI is the relationship between diastolic and systolic ambulatory blood pressure (BP) over 24-hours, which can be obtained by performing a linear regression analysis of diastolic BP over systolic BP and subtracting the slope from $1 .{ }^{8}$ Cardiovascular risk, in particular stroke, was predicted by AASI in different adult populations. ${ }^{9,10}$ In childhood, increased AASI has been stated in children with hypertension and diabetes mellitus. ${ }^{11}$ Besides AASI, ambulatory blood pressure monitoring can be used to calculate pulse pressure and these two parameters have been suggested as markers of arterial stiffness and predictors of cardiovascular disease. ${ }^{11}$ To assess arterial stiffness, few studies have investigated AASI in obese children. ${ }^{12}$

In this prospective trial, we have studied the difference of AASI between obese and lean children.

\section{Material and Methods}

\section{Study population}

In this prospective cohort 53 obese children (33 girls) aged between 4 -16 years were investigated at pediatric outpatient clinic, Ministry of Health Bağcllar Training and Research Hospital, Istanbul, Turkey. The control group consisted of age-matched 42 healthy subjects (20 girls) aged between 5-18 years. Obesity was defined as body mass index $(\mathrm{BMI}) \geq 95^{\text {th }}$ percentile according to height- and sex-specific BMI charts by Centers for Disease Control and Prevention (CDC). ${ }^{13}$ The control group included lean children with a normal BMI percentile $\left(<85^{\text {th }}\right.$ percentile). Weight and height were measured and recorded in all subjects.

Informed consent was obtained from all individual participants (and/or their parents or legal guardians) included in the study.

\section{Laboratory evaluation}

In the obese group blood samples were collected in the morning after an overnight fast for the assessment of metabolic parameters which included fasting glucose level, total cholesterol, low-density lipoprotein (LDL) cholesterol, high-density lipoprotein (HDL) cholesterol, triglycerides, insulin, 25-hydroxyvitamin D [25(OH)D], and high sensitive C-reactive protein (hsCRP). Homeostasis model assessment of insulin resistance (HOMA-IR) was calculated using the equation HOMA-IR = [Fasting plasma insulin $(\mathrm{miU} / \mathrm{mL}) \times$ Fasting plasma glucose $(\mathrm{mg} /$ dl)] / 405. HOMA-IR cut-off values for insulin resistance in the prepubertal period were accepted to be 2.67 in boys and 2.22 in girls, and in the pubertal period, 5.22 in boys and 3.82 in girls. ${ }^{14}$ Dyslipidemia was defined as serum levels of total cholesterol $\geq 200 \mathrm{mg} / \mathrm{dl}$ and/or LDL $\geq 130 \mathrm{mg} / \mathrm{dl}$ and/or triglycerides $\geq 100 \mathrm{mg} /$ $\mathrm{dl}$ in the 6-9 years of age group and $\geq 130 \mathrm{mg} /$ $\mathrm{dl}$ in the 10-18 years of age group according to the National Heart, Lung, and Blood Institute (NHLBI) criteria. $^{15}$ Vitamin D deficiency was defined as $<30 \mathrm{nmol} / \mathrm{L} .{ }^{16}$ The normal limits of hsCRP were accepted as $0-5 \mathrm{mg} / \mathrm{dl}$.

\section{Blood pressure assessments}

Casual blood pressure (BP) measurement was performed three times with 10 min intervals by an oscillometric device (Nihon Kohden, Vismo, Germany) and was averaged; indexed systolic (SBP) and diastolic (DBP) BP were calculated by dividing $95^{\text {th }}$ percentile according to height and gender. ${ }^{17}$ 24-hour ambulatory blood pressure monitoring (ABPM) was performed by using a portable device (SpaceLabs 90217A1 oscillometric device, Spacelabs Healthcare, UK). We used different cuff sizes according to the patient's arm circumference. Width of the inflatable bladder of the cuff was chosen as $40 \%$ of upper arm circumference, and length of the inflatable bladder was decided as $80 \%$ of upper arm circumference (almost long enough to encircle the arm). The non-dominant arm served for cuff placing. Blood pressure recordings were obtained with 20-min intervals in the wake 
period and 30-min intervals in the sleep period. Keeping a diary (particularly sleep disturbances) was asked to participants for evaluating daytime and nighttime BP measurements. ABPM recordings were considered sufficient if it contained $\geq 75 \%$ successful measurements. Patients with insufficient (less than $75 \%$ ) blood pressure measurements (i.e., due to the manual application of the device or a software problem) were excluded from the study. Hypertension was defined according to the criteria of American Heart Association (AHA). ${ }^{18}$

\section{Ambulatory arterial stiffness index}

Twenty-four-hour blood pressure measurements were entered into the Statistical Package for the Social Sciences (SPSS) software program (IBM SPSS Statistics, Armonk, NY) for each patient. Linear regression was performed on the 24-hour BP recordings, with diastolic BP as the dependent variable and the systolic BP as the independent variable. The unstandardized regression slope (B) was considered in this study since the 24-hour BP recordings were unchanged. AASI was calculated by subtracting the regression slope from 1 .

Other parameters that were recorded from the BP device included the 24-hour, day and night heart rates, systolic and diastolic BP values and loads, and pulse pressures (PP). Standard deviation score (SDS) was calculated for the mean 24-hour, day and night heart rate, systolic and diastolic BP values by the LMS method using height-specific normative values [degree of skewness (L), median (M) and coefficient of variation $(S)] \cdot{ }^{19}$

All procedures performed in studies involving human participants were in accordance with the ethical standards of the institutional research committee at which the studies were conducted (Ministry of Health Bağcılar Training and Research Hospital, approval number 2015/366) and with the 1964 Helsinki declaration and its later amendments or comparable ethical standards.

\section{Statistical analysis}

Data analysis was carried out using SPSS software version 21 (IBM SPSS Statistics, Armonk, NY). All parameters were examined in terms of distribution of parameters by the adjusted Fisher-Pearson standardized moment coefficient. Continuous data were expressed as the mean $\pm \mathrm{SD}$ if the distribution was normal and/or median (IQR) otherwise. Student- $t$ test was used for the comparison of normally distributed variables, and Mann-Whitney U test was used if data was not normally distributed. Categorical variables were presented as a number and compared using the Chi-squared test and the Fisher's exact test. Associations among AASI, PP, and other parameters were assessed by Pearson correlation analysis if data was normally distributed and by Spearman correlation analysis if data was not normally distributed. The variables that showed a $p$ value of $<0.20$ in the univariate analysis and parameters which were reported to be associated in the previous studies were tested in a multivariate linear regression analysis to identify the important independent factors influencing AASI values. The abnormally distributed parameters were transformed into a normal distribution by logarithmic transformation. Significance was allowed at $p$ $<0.05$.

\section{Results}

Demographic features of obese and healthy groups are summarized in Table I. Weight and BMI-SDS of the obese group were significantly higher than the healthy group $(p<0.001)$. Metabolic features of obese participants are presented in Table II. Dyslipidemia was determined in 21 obese children (39.6\%) [including increased total cholesterol (n: 1), increased LDL-cholesterol (n: 5), increased triglycerides levels (n: 11), at least two abnormal lipid values (n: 2), and at least three abnormal lipid levels (n: 2)]. Increased HOMA-IR rate was calculated in 30 obese children (56.6\%). A total of 47 obese children $(88.7 \%)$ were described 
Table I. Characteristics of healthy and obese participants.

\begin{tabular}{|c|c|c|c|}
\hline Characteristics & $\begin{array}{l}\text { Healthy participants } \\
\text { (n: } 42)\end{array}$ & $\begin{array}{l}\text { Obese participants } \\
\text { (n: } 53)\end{array}$ & $p$ value \\
\hline Age, years & $11.3 \pm 3.17$ & $10.6 \pm 2.83$ & 0.48 \\
\hline Male/Female, n (\%) & $22(52) / 20(48)$ & $20(37) / 33(63)$ & 0.15 \\
\hline Height, m & $1.50 \pm 0.17$ & $1.46 \pm 0.16$ & 0.36 \\
\hline Height-SDS & $0.51 \pm 1.41$ & $0.63 \pm 1.04$ & 0.57 \\
\hline Weight, kg & $45.5 \pm 15.1$ & $65.0 \pm 21.8$ & $<0.001$ \\
\hline Weight-SDS & $0.42 \pm 1.17$ & $2.86 \pm 0.83$ & $<0.001$ \\
\hline BMI, kg/m² & $19.6 \pm 3.49$ & $29.1 \pm 4.42$ & $<0.001$ \\
\hline BMI-SDS & $0.23 \pm 1.03$ & $2.71 \pm 0.57$ & $<0.001$ \\
\hline
\end{tabular}

BMI: body mass index, SDS: standard deviation score.

Table II. Metabolic features of obese participants.

\begin{tabular}{lc}
\hline Features & $\begin{array}{c}\text { Obese participants } \\
\text { (n: } 53)\end{array}$ \\
\hline eGFR, ml/min/1.73m²* & $120(21)$ \\
Total cholesterol, mg/dl & $162.1 \pm 26.9$ \\
LDL-C, mg/dl & $90.1 \pm 26.1$ \\
HDL-C, mg/dl & $53.2 \pm 17.1$ \\
Triglycerides, mg/dl & $101(80.5)$ \\
Fasting glucose, mg/dl & $90.2 \pm 9.9$ \\
Insulin, miU/ml* & $17.5(15)$ \\
HOMA-IR* & $3.69(3.87)$ \\
Vitamin D, ng/ml* & $17.8(12.6)$ \\
hsCRP, mg/L* & $1.05(1.17)$ \\
\hline
\end{tabular}

eGFR: estimated glomerular filtration rate, LDL-C: lowdensity lipoprotein cholesterol, HDL-C: high-density lipoprotein cholesterol, hsCRP: high sensitive $\mathrm{C}$ reactive protein, HOMA-IR: homeostasis model assessment of insulin resistance.

* Non-parametric values are presented as median (IQR).

as vitamin D deficient. Increased hsCRP level $(>5 \mathrm{mg} / \mathrm{dl})$ was found in five obese children $(9.4 \%)$.

Hypertension was found in three obese participants (5.6\%) including masked HT (n: 2) and ambulatory HT (n: 1). Five obese children had prehypertension and 17 had white coat hypertension. All subjects were normotensive in the healthy group. Indexed casual SBP and DBP, 24-hour, daytime and nighttime SBP-SDS, DBP-SDS, and MAP-SDS did not significantly differ between the obese and healthy groups. Although a statistically significant difference was found in the mean value of heart rate between the groups, there was no significant between-group difference in the median values of 24-h, day- and nighttime heart rate-SDS (Table III).

While the mean pulse pressure values did not significantly differ between the groups, the mean AASI was significantly higher in obese children compared to healthy subjects (0.42 \pm 0.15 versus $0.29 \pm 0.18, p<0.001$; Table III). The clinical parameters (age, gender, heightSDS and BMI-SDS) blood pressure parameters (logarithmic-indexed-casual SBP and DBP, 24-h, MAP-SDS, nighttime SBP-SDS and load, systolic and diastolic nocturnal dipping) and laboratory findings (vitamin D and LDL,cholesterol, levels) were separately analyzed in the obese and healthy subjects by the univariate analysis to determine the factors influencing AASI. All variables with a $p$ value of $<0.20$ and parameters which were reported to be associated in previous studies (age, gender, height-SDS, BMI-SDS, log-indexed-casual SBP, nighttime SBP-SDS, nighttime SBP-Load, systolic and diastolic nocturnal dipping) were included in the enter model of the multivariate linear regression analyses. As shown in Table IV, AASI significantly correlated with indexedcasual SBP, nighttime SBP-SDS and nighttime SBP load, systolic and diastolic nocturnal dipping, with no independent predictor. Furthermore, AASI was evaluated with the presence of dyslipidemia, increased HOMAIR and metabolic abnormality, no significant difference was noted. 
Table III. Results of arterial stiffness indexes, casual and 24-h ambulatory blood pressure measurements of participants.

\begin{tabular}{lccc}
\hline Measurements & $\begin{array}{c}\text { Healthy participants } \\
\mathrm{n}=42\end{array}$ & $\begin{array}{c}\text { Obese participants } \\
\mathrm{n}=53\end{array}$ & $p$ value \\
\hline Indexes of arterial stiffness & & & \\
$\quad$ AASI & $0.29 \pm 0.18$ & $0.42 \pm 0.15$ & $<0.001$ \\
$\quad$ Pulse pressure, mmHg* & $44.5(9)$ & $44(10)$ & 0.23 \\
24-h HR, bpm & $83.2 \pm 8.4$ & $88.2 \pm 7.5$ & 0.002 \\
24-h HR-SDS * & $0.10(0.01)$ & $0.10(0.00)$ & 0.48 \\
$\quad$ Daytime HR-SDS * & $-0.50(1.00)$ & $-0.10(1.06)$ & 0.058 \\
$\quad$ Nighttime HR-SDS * & $0.21(1.55)$ & $0.60(1.15)$ & 0.46 \\
Casual SBP, indexed & $0.91 \pm 0.08$ & $0.95 \pm 0.10$ & 0.73 \\
Casual DBP, indexed & $0.81 \pm 0.08$ & $0.84 \pm 0.12$ & 0.27 \\
24-h SBP-SDS & $-0.15 \pm 0.92$ & $-0.24 \pm 1.15$ & 0.54 \\
24-h SBP-load, \% * & $5.5(12.0)$ & $7.0(18.0)$ & 0.80 \\
24-h DBP-SDS & $-0.28 \pm 0.83$ & $-0.63 \pm 0.99$ & 0.73 \\
24-h DBP-load, \% * & $8.0(10.0)$ & $9.0(16.0)$ & 0.39 \\
24-h MAP-SDS & $0.06 \pm 0.77$ & $0.02 \pm 0.90$ & 0.72 \\
Daytime SBP-SDS & $-0.45 \pm 0.93$ & $-0.49 \pm 1.06$ & 0.27 \\
Daytime SBP-load, \% & $3.5(9.9)$ & $6.9(13.7)$ & 0.59 \\
Daytime DBP-SDS* & $-0.86(1.00)$ & $-0.60(1.23)$ & 0.85 \\
Daytime DBP-load, \% * & $5.2(9.1)$ & $7.1(12.1)$ & 0.08 \\
Nighttime SBP-SDS & $0.27 \pm 0.79$ & $0.32 \pm 1.12$ & 0.12 \\
Nighttime SBP- load, \% * & $10.2(29.6)$ & $10.0(31.6)$ & 0.53 \\
Nighttime DBP-SDS* & $0.61(0.98)$ & $0.21(1.44)$ & 0.051 \\
Nighttime DBP- load, \% * & $13.8(27.6)$ & $12.5(31.6)$ & 0.83 \\
Non-dippers for SBP (n, \%) & $33(78.6)$ & $34(64.2)$ & 0.12 \\
Non-dippers for DBP, $\mathrm{n}$ (\%) & $15(35.7)$ & $12(22.6)$ & 0.13 \\
\hline AASt: & & & \\
\hline
\end{tabular}

AASI: ambulatory arterial stiffness index, DBP: diastolic blood pressure, HR: heart rate, MAP: mean arterial pressure, SBP: systolic blood pressure, SDS: standard deviation score, NS: not significant.

* Non-parametric values are presented as median (IQR).

\section{Discussion}

The important finding of this study was that the AASI was significantly increased in obese children, with no significant difference in the pulse pressure.

Arterialstiffnesshas beennoted as one of themost important hemodynamic factors contributing to the development of cardiovascular complications. ${ }^{20}$ Increased arterial stiffness can predict the risk for cardiovascular death and development of cardiovascular disease at an early stage before vascular lesions induce symptoms. ${ }^{21}$ The last decade has observed increased interest in the methods of measuring arterial elasticity, either directly or by the way of surrogate measures. The important question that needs to be answered is which methods are the best options for assessing arterial stiffness. The answer should include cost-effective, accessible, and easy methods.

The most common available techniques for evaluating arterial stiffness are those measuring carotid artery IMT (with M-mode measurements), flow-mediated dilatation, PWV and augmentation index. These methods have been shown to predict cardiovascular events in patients with hypertension, kidney failure, 
Table IV. Univariate and multivariate analysis of AASI, clinical and laboratory parameters of obese participants.

\begin{tabular}{lccccc}
\hline \multirow{2}{*}{ Parameters } & \multicolumn{2}{c}{ Univariate analysis } & \multicolumn{3}{c}{ Multivariate analysis } \\
\cline { 2 - 6 } & Correlation $r$ & $p$ value & $95 \%$ CI & Beta value & $p$ value \\
\hline Age & 0.018 & 0.86 & -0.016 to 0.012 & -0.034 & 0.80 \\
Gender & -0.196 & 0.80 & -0.151 to 0.023 & -0.205 & 0.14 \\
Height-SDS & -0.088 & 0.40 & -0.021 to 0.055 & 0.115 & 0.37 \\
BMI-SDS & -0.122 & 0.39 & -0.093 to 0.048 & -0.084 & 0.52 \\
Log-indexed-casual SBP & 0.251 & 0.07 & -0.049 to 1.561 & 0.247 & 0.065 \\
Log-indexed-casual DBP & -0.012 & 0.93 & & & \\
24-h MAP-SDS & 0.023 & 0.82 & & & \\
Nighttime SBP-SDS & 0.220 & 0.032 & -0.066 to 0.057 & -0.033 & 0.88 \\
Nighttime SBP-load & 0.199 & 0.068 & -0.001 to 0.004 & 0.215 & 0.29 \\
Systolic nocturnal dipping & -0.335 & 0.001 & -0.019 to -0.001 & -0.356 & 0.074 \\
Diastolic nocturnal dipping & -0.271 & 0.008 & -0.007 to 0.004 & -0.133 & 0.41 \\
LDL-C & 0.031 & 0.82 & & & \\
Vitamin-D & 0.022 & 0.87 & & & \\
\hline
\end{tabular}

CI: confidence interval, DBP: diastolic blood pressure, LDL-C: low-density lipoprotein cholesterol; Log: logarithm; MAP: mean arterial pressure, SBP: systolic blood pressure, SDS: standard deviation score.

and diabetes mellitus. ${ }^{22,23}$ However, all these techniques require experienced persons and expensive tools. AASI, which is an accessible, cost-effective, and easy method derived from ABPM, was introduced as an index that predicts cardiovascular risk and particularly stroke in different populations. ${ }^{8,9}$ In some studies, AASI has been found to be correlated with PWV and carotid-IMT. ${ }^{24}$ However, some researchers have found only a weak correlation between AASI and PWV and have suggested AASI only as a surrogate measure of arterial stiffness. ${ }^{25}$ Until now there has been no study to validate AASI and other measurement techniques of arterial stiffness. Increased AASI has been previously shown in patients with hypertension, diabetes mellitus, and end-stage kidney disease (ESKD). ${ }^{11,26,27}$ In the obese population, AASI has been investigated in very few studies and was found to be significantly higher. ${ }^{12,28}$ Similarly, the important finding in our study was that AASI increased significantly in obese children compared to matched lean children.

Over the last decade, researchers have focused on measures of subclinical atherosclerosis in childhood obesity to predict cardiovascular risk in adulthood. In previous studies, it has been shown that adolescents whose LDL-cholesterol levels were elevated, showed increased carotid artery IMT and endothelial dysfunction. Obesity itself, along with insulin resistance, dyslipidemia, and hypertension, may contribute to increased cardiovascular morbidity and mortality. ${ }^{13}$ In our study dyslipidemia was noted in $39.6 \%$ of the obese children, with no significant correlation between AASI and LDLcholesterol. A greater number of patients are needed to assess this relationship.

Pulse pressure is another marker to evaluate arterial stiffness. In previous studies, pulse pressure was assessed together with AASI and found to be significantly increased in hypertensive obese population. ${ }^{12,29}$ Also, increased pulse pressure has been suggested to be related with aging and decreased vascular compliance. ${ }^{30}$ On the contrary, there was no significant difference in pulse pressure between obese and lean children in our study. It may be associated with the younger age and lower rate of hypertension in our participants.

Previously, AASI was studied in a large group of hypertensive adults and was determined to be significantly increased. ${ }^{8,9}$ The data on AASI in children with hypertension are insufficient. 
Some researchers reported that hypertension was the most important and independent factor affecting AASI. ${ }^{12}$ Simonetti et al. $^{26}$ showed that hypertensive children had an increased AASI regardless of their BMI. On the contrary, Stergiou et al..$^{29}$ stated a significant relationship between AASI and BMI with no correlation to hypertension. Our study which included obese children who were nearly all normotensive showed no significant correlation between AASI and BMI-SDS. On the other hand, AASI significantly correlated with indexed-casual SBP, nighttime SBP-SDS and -load, systolic and diastolic nocturnal dipping, with no independent predictor. The cause of increased AASI in normotensive obese individuals needs to be clarified. We do not have an accurate explanation concerning increased arterial stiffness in normotensive obese population. Some theories have been asserted to explain the causes of increased AASI including inflammation, oxidative stress, and activation of the sympathetic nervous system. In our study, $94 \%$ of the participants were normotensive, and $9.4 \%$ had elevated hsCRP levels which were measured as an inflammatory marker. In univariate correlation analysis, AASI was not associated with hsCRP.

The strength of this study comes from the evaluation of mostly normotensive obese children and the comparison to a matched control group of lean, healthy children. Furthermore, it was essential to show the significant correlation between AASI and indexed-casual SBP, nighttime SBP-SDS and load, systolic and diastolic nocturnal dipping.

The limitations of this study includes a low sample size, lack of waist circumference, lack of correlation with PWV and carotid distensibility, and lack of other inflammation markers.

In conclusion, this study confirms that AASI increased significantly even in normotensive obese children and that AASI is mainly influenced by systolic BP. This method is a cheaper, easier, and a more accessible method than others to assess arterial stiffness. Further prospective studies are needed to explain the causes of increased AASI in normotensive populations, to investigate the role of inflammation, oxidative stress, and activation of the sympathetic nervous system, and to evaluate the association between AASI and cardiovascular disease. Early detection of increased arterial stiffness can help clinicians come up with preventive measures in the management of their patients.

\section{Acknowledgments}

We thank the patients and their families taking part in this study.

\section{REFERENCES}

1. World Obesity Federation, World Map of Obesity. Available at: http://www.worldobesity.org/ aboutobesity/world-map-obesity/?map=children. (Accessed on April 2, 2017)

2. Rosner B, Cook NR, Daniels S, Falkner B. Childhood blood pressure trends and risk factors for high blood pressure: The NHANES experience 1988-2008. Hypertension 2013; 62: 247-254.

3. Theodore RF, Broadbent J, Nagin D, et al. Childhood to early-midlife systolic blood pressure trajectories: Early-life predictors, effect modifiers, and adult cardiovascular outcomes. Hypertension 2015; 66: 1108-1115.

4. Berenson GS, Srinivasan SR, Bao W, Newman WP, 3rd, Tracy RE, Wattigney WA. Association between multiple cardiovascular risk factors and atherosclerosis in children and young adults. The Bogalusa Heart Study. N Engl J Med 1998; 338: 16501656.

5. Beauloye V, Zech F, Tran HT, Clapuyt P, Maes M, Brichard SM. Determinants of early atherosclerosis in obese children and adolescents. J Clin Endocrinol Metab 2007; 92: 3025-3032.

6. Skilton MR, Celermajer DS. Endothelial dysfunction and arterial abnormalities in childhood obesity. Int J Obes (Lond) 2006; 30: 1041-1049.

7. Aggoun $\mathrm{Y}$, Farpour-Lambert NJ, Marchand LM, Golay E, Maggio AB, Beghetti M. Impaired endothelial and smooth muscle functions and arterial stiffness appear before puberty in obese children and are associated with elevated ambulatory blood pressure. Eur Heart J 2008; 29: 792-799. 
8. Dolan E, Li Y, Thijs L, et al. Ambulatory arterial stiffness index: rationale and methodology. Blood Press Monit 2006; 11: 103-105.

9. Dolan E, Thijs L, Li Y, et al. Ambulatory arterial stiffness index as a predictor of cardiovascular mortality in the Dublin Outcome Study. Hypertension 2006; 47: 365-370.

10. Kikuya M, Staessen JA, Ohkubo T, et al. Ambulatory arterial stiffness index and 24-hour ambulatory pulse pressure as predictors of mortality in Ohasama, Japan. Stroke 2007; 38: 1161-1166.

11. Sulakova T, Janda J, Cerna J, Janstova V, Feber J. Assessment of arterial stiffness from ambulatory blood pressure monitoring in children with diabetes mellitus type-1 (DMT1). J Hum Hypertens 2012; 26: 357-364.

12. Saner C, Simonetti GD, Wuhl E, Mullis PE, Janner $M$. Increased ambulatory arterial stiffness index in obese children. Atherosclerosis 2015; 238: 185-189.

13. Kuczmarski RJ, Ogden CL, Guo SS, et al. 2000 CDC growth charts for the United States: methods and development. National Center for Health Statistics. Vital Health Stat 11 2002; 246: 1-190.

14. Kurtoğlu S, Hatipoğlu N, Mazıcıoğlu M, Kendirici M, Keskin M, Kondolot M. Insulin resistance in obese children and adolescents: HOMA-IR cut-off levels in the prepubertal and pubertal periods. J Clin Res Pediatr Endocrinol 2010; 2: 100-106.

15. Expert Panel on Integrated Guidelines for Cardiovascular Health and Risk Reduction in Children and Adolescents; National Heart, Lung and Blood, Institute. Expert panel on integrated guidelines for cardiovascular health and risk reduction in children and adolescents: summary report. Pediatrics 2011; 128(Suppl 5): S213-S256.

16. Munns CF, Shaw N, Kiely M, et al. Global Consensus Recommendations on Prevention and Management of Nutritional Rickets. J Clin Endocrinol Metab 2016; 101: 394-415.

17. Neuhauser HK, Thamm M, Ellert U, Hense HW, Rosario AS. Blood pressure percentiles by age and height from nonoverweight children and adolescents in Germany. Pediatrics 2011; 127: e978-e988.

18. Flynn JT, Daniels SR, Hayman LL, et al. Update: ambulatory blood pressure monitoring in children and adolescents: a scientific statement from the American Heart Association. Hypertension 2014; 63: 1116-1135.

19. Wuhl E, Witte K, Soergel M, Mehls O, Schaefer F; German Working Group on Pediatric Hypertension. Distribution of 24-h ambulatory blood pressure in children: normalized reference values and role of body dimensions. J Hypertens 2002; 20: 1995-2007.
20. Laurent S, Cockcroft J, Van Bortel L, et al; European Network for Non-invasive Investigation of Large Arteries. Expert consensus document on arterial stiffness: methodological issues and clinical applications. Eur Heart J 2006; 27: 2588-2605.

21. Domanski M, Norman J, Wolz M, Mitchell G, Pfeffer M. Cardiovascular risk assessment using pulse pressure in the first national health and nutrition examination survey (NHANES I). Hypertension 2001; 38: 793-797.

22. Mancia G, De Backer G, Dominiczak A, et al; Management of Arterial Hypertension of the European Society of Hypertension; European Society of Cardiology. 2007 Guidelines for the Management of Arterial Hypertension: The Task Force for the Management of Arterial Hypertension of the European Society of Hypertension (ESH) and of the European Society of Cardiology (ESC). J Hypertens 2007; 25: 1105-1187.

23. Wang KL, Cheng HM, Sung SH, et al. Wave reflection and arterial stiffness in the prediction of 15-year allcause and cardiovascular mortalities: a communitybased study. Hypertension 2010; 55: 799-805.

24. Li Y, Wang JG, Dolan E, et al. Ambulatory arterial stiffness index derived from 24-hour ambulatory blood pressure monitoring. Hypertension 2006; 47: 359-364.

25. Leoncini G, Ratto E, Viazzi F, et al. Increased ambulatory arterial stiffness index is associated with target organ damage in primary hypertension. Hypertension 2006; 48: 397-403.

26. Simonetti GD, Von Vigier RO, Wuhl E, Mohaupt MG. Ambulatory arterial stiffness index is increased in hypertensive childhood disease. Pediatr Res 2008; 64: 303-307.

27. Degi A, Kerti A, Cseprekal O, et al. Ambulatory arterial stiffness index in children after kidney transplantation. Pediatr Transplant 2013; 17: 598604.

28. Prochotska K, Kovacs L, Vitariusova E, Feber J. Is arterial stiffness predicted by continuous metabolic syndrome score in obese children? J Am Soc Hypertens 2016; 10: 47-54.

29. Stergiou GS, Kollias A, Giovas PP, Papagiannis J, Roussias LG. Ambulatory arterial stiffness index, pulse pressure and pulse wave velocity in children and adolescents. Hypertens Res 2010; 33: 1272-1277. 